\title{
CARACTERIZAÇÃO ESTRUTURAL E MECÂNICA DE FIOS ORTODÔNTICOS DE NiTi*
}

\author{
Pedro Damas Resende 1 \\ Allan Souza Portes ${ }^{2}$ \\ Natália Isabel Azevedo Lopes ${ }^{3}$ \\ Leandro de Arruda Santos ${ }^{4}$ \\ Ana Cristina Rodrigues Antunes de Souza ${ }^{5}$ \\ Vicente Tadeu Lopes Buono ${ }^{6}$
}

\section{Resumo}

Fios ortodônticos de NiTi são alternativas para substituição dos materiais convencionais para a mesma utilização. Quando comparado ao aço inoxidável, NiTi apresenta resistência à corrosão semelhante e características mecânicas superiores, uma vez que produz forças de menor intensidade e constantes numa grande faixa de deformação. Foram caracterizados grupos de fios ortodônticos de dois fabricantes, com diferenças no tipo de ativação e em seus diâmetros. Foram utilizadas microscopia eletrônica de varredura e espectroscopia de energia dispersiva para caracterização superficial e de composição dos fios. As fases presentes foram avaliadas por difração de raios $\mathrm{X} e$ ensaios mecânicos de dobramento de três pontos e tração foram conduzidos de forma a avaliar suas propriedades mecânicas. Todas as amostras apresentaram conteúdo de níquel acima de 50,5 at.\%. Os fios superelásticos apresentaram recuperação total de forma em ambos os testes mecânicos e os fios termoativados apresentaram recuperação parcial de forma. Os difratogramas obtidos para todos os grupos apresentaram apenas fase austenítica anteriormente ao carregamento. Todos os fios superelásticos apresentaram maior força no descarregamento quando comparados com os termoativados.

Palavras-chave: Fios ortodônticos; NiTi; Superelasticidade, Memória de forma.

\section{MECHANICAL AND STRUCTURAL CHARACTERIZATION OF ORTHODONTIC ARCHWIRES}

\section{Abstract}

Nickel-titanium orthodontic archwires are alternatively used instead of conventional materials applied to the same purpose. When compared with stainless steel, NiTi alloy presents similar corrosion resistance and superior mechanical properties, since undergoes constant and less intense forces under large deformations. Groups of orthodontic archwires manufactured by two different companies were assessed by means of SEM to evaluate the surface of the wires and its semi-quantitative atomic compositions (EDS). The present phases were evaluated by XRD and mechanical tests were performed in a three-point bending testing machine and by uniaxial tensile tests. It was found that the content of nickel on the material is above 50.5 at.\%. All the superelastic wires presented full shape recovery in both mechanical tests. The thermoactivated wires exhibited partial recovery of deformation on both tests, which means that superelasticity and shape memory effect coexist in the material. The XRD analysis has shown only austenitic phase on both groups. All the groups of superelastic wires have higher forces during unloading, when compared to the thermoactivated.

Keywords: Orthodontic archwires; NiTi; Superelasticity; Shape mamory effect.

1 Graduando do curso de Engenharia Metalúrgica, Universidade Federal de Minas Gerais (UFMG), Belo Horizonte (BH), Minas Gerais (MG), Brasil.

2 Dentista, Mestrando do Programa de Pós-Graduação em Engenharia Metalúrgica, Materiais e de Minas, Escola de Engenharia, UFMG, Belo Horizonte, MG, Brasil

3 Engenheira Química, Doutoranda do Programa de Pós-graduação em Engenharia Metalúrgica, Materiais e Minas, Escola de Engenharia, UFMG, Belo Horizonte, MG, Brasil.

4 Eng. de Materiais, doutor, Professor Auxiliar, Depto. Eng. Metalúrgica e de Materiais, UFMG, BH, MG, Brasil.

5 Dentista, MSc, Dr., Professora Adjunta, Centro Universitário Newton Paiva, Belo Horizonte, MG, Brasil

6 Físico, Doutor, Professor Titular, Depto. Eng. Metalúrgica e de Materiais, UFMG, BH, MG, Brasil. 


\section{INTRODUÇÃO}

Ligas NiTi são conhecidas por sua vasta utilização como materiais funcionais e biomateriais. O que as diferenciam das demais ligas comerciais são suas propriedades mecânicas únicas e biocompatibilidade. Quando sua composição se aproxima da composição equiatômica, podendo haver um leve excesso de níquel, o NiTi apresenta o chamado efeito memória de forma (EMF). Transformações de fase adifusionais no estado sólido são responsáveis por tal efeito. Inicialmente, a matriz do material se encontra num estado em que a fase estável é a austenítica, caracterizada por uma estrutura do tipo B2. Ao promover o resfriamento progressivo a partir desta fase atinge-se uma temperatura em que a transformação direta, da austenita para martensita, se inicia (Ms). A temperatura a partir da qual não se pode detectar nenhuma fração da fase inicial marca o fim da transformação direta (Mf). Resultando numa estrutura martensítica do tipo B19'. A transformação no caminho inverso, dita reversa, acontece com aquecimento do material, porém com temperaturas de início e fim de transformação martensita - austenita (As e Af) não coincidentes com as temperaturas exibidas no resfriamento [1]. Há também possibilidade de que haja uma estrutura intermediária na transformação, conhecida como fase $R$, que também apresenta suas devidas temperaturas de início e fim de transformação (Rs e Rf)[2].

A transformação martensítica em ligas com memória de forma também pode ser induzida por tensão ao se aplicar um carregamento mecânico ao material. O comportamento em tração uniaxial de ligas NiTi com EMF é bem característico, parte-se de um corpo de prova completamente austenítico, em que se deforma elasticamente a fase B2 inicial até que se atinge uma tensão crítica de indução de martensita $\left(\sigma_{c}\right)$, com a progressão do carregamento há um patamar de tensão associado à transformação martensítica que chega a acomodar valores de deformação da ordem de 6\%. Quando o material está completamente transformado inicia-se a deformação elástica da martensita e com a evolução da deformação atinge-se o limite de escoamento desta fase, iniciando-se o regime de deformação plástica, que apresenta pouca ductilidade, até que se rompe o corpo de prova. Quando a deformação ocorre sob temperaturas acima de Af, a fase austenítica é estável e a transformação reversa acontece espontaneamente e fica caracterizada a superelasticidade (SE)[1-3].

Os limites em que a ruptura do material deve ser considerada são pouco relevantes para a utilização destas ligas. De forma geral, as aplicações das ligas NiTi, que apresentam EMF, se beneficiam do fato do material poder acomodar grandes deformações e retornar ao seu estado inicial, anterior ao carregamento. Quando se avalia uma curva tensão-deformação abaixo do limite de escoamento da martensita, a recuperação da deformação segue um padrão semelhante ao do carregamento, exceto pelo fato de que o patamar de transformação reversa exibe valores abaixo do patamar da transformação direta, como evidenciado na figura1. 


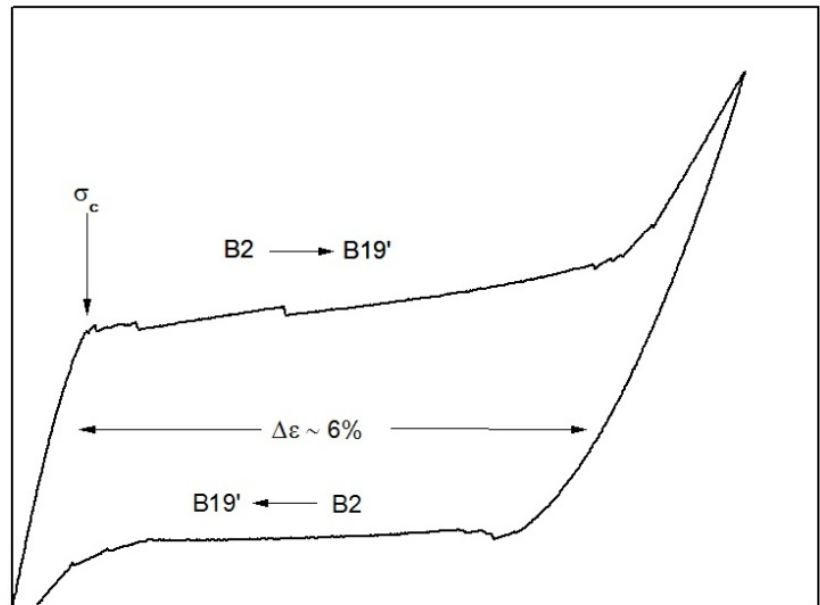

Figura 1 - Ciclo de carga e descarga em tração de NiTi.

A utilização da liga NiTi na ortodontia se iniciou na década de 1970 quando a primeira liga $\mathrm{Ni} 50 \%$ at - Ti foi introduzida no mercado. Inicialmente a liga utilizada não apresentava SE ou EMF, uma vez que os efeitos eram suprimidos no processo de obtenção das ligas, devido ao alto grau de encruamento proveniente da conformação do material [4]. Parte-se do princípio que o material que promove um bom movimento dentário possui baixa força de retorno no descarregamento e continuidade desta força [4,5]. A fase martensítica era estabilizada devido à deformação plástica e sua utilização para tratamentos ortodônticos se demonstrava interessante, uma vez que o módulo de elasticidade da martensita é bem menor que o dos aços inoxidáveis usados anteriormente.

Atualmente os fios de NiTi são divididos pelo tipo de ativação do movimento dentário que apresentam em tratamento. Há os fios com ativação superelástica, aqueles que são deformados e encaixados aos bráquetes acima de sua temperatura Af. Assim, a transformação reversa será espontânea e ocorrerá independentemente da temperatura bucal [4]. Existem também os fios de ativação térmica (ou termoativados), estes são deformados para que se encaixem nos bráquetes em sua forma de baixa temperatura, ou seja, abaixo de Af, o que faz com que não aconteça a transformação reversa imediatamente. Uma vez o fio colocado, a temperatura bucal é suficiente para que Af seja excedida, promovendo, assim, a transformação reversa e por consequência a movimentação dentária.

Este trabalho tem como objetivo avaliar o comportamento mecânico de diferentes fios ortodônticos e relacioná-lo com o desempenho clínico. Para tal, caracterizou-se a superfície de diferentes fios ortodônticos de $\mathrm{NiTi}$, bem como avaliou-se as propriedades mecânicas através de ensaios de dobramento. Foram realizadas também difração de raios $X(D R X)$ e espectroscopia de energia dispersiva (EDS).

\section{MATERIAIS E MÉTODOS}

Foram caracterizados fios ortodônticos comerciais de NiTi pré-moldados no formato da arcada dentária. Fios no estado superelástico e termoativados de dois fabricantes diferentes foram testados com dois diâmetros distintos, totalizando oito grupos de amostra, resumidos na tabela 1 . 
Tabela 1 - Descrição das amostras analisadas

\begin{tabular}{|c|c|c|c|}
\hline Fabricante & Tipo de ativação & Diâmetro (in) & Nome da amostra \\
\hline \multirow{4}{*}{ 3M Unitek } & \multirow{2}{*}{ Superelástico } & 0,018 & 3M SE 18 \\
\hline & & 0,016 & 3 M SE 16 \\
\hline & \multirow{2}{*}{ Termoativado } & 0,018 & 3M TA 18 \\
\hline & & 0,016 & 3M TA 16 \\
\hline \multirow{4}{*}{$\begin{array}{l}\text { American } \\
\text { Orthodontics }\end{array}$} & \multirow{2}{*}{ Superelástico } & 0,018 & AO SE 18 \\
\hline & & 0,016 & AO SE 16 \\
\hline & \multirow{2}{*}{ Termoativado } & 0,018 & AO SE 18 \\
\hline & & 0,016 & AO SE 16 \\
\hline
\end{tabular}

A superfície dos fios foi avaliada qualitativamente por microscopia eletrônica de varredura (FEI Inspect, F50) operando a $15 \mathrm{kV}$ e obtendo-se imagens de elétrons secundários. No mesmo equipamento obteve-se uma análise química semiquantitativa por EDS.

A caracterização mecânica foi realizada por meio de ensaio de dobramento de três pontos (AN8032, Analógica Instrumentação e Controle, Belo Horizonte, MG) com corpo de prova de $30 \mathrm{~mm}$ de comprimento, distância entre os apoios de $10 \mathrm{~mm}$ e velocidade do braço da máquina de $1,3 \mathrm{~mm} \cdot \mathrm{min}^{-1}$. Foram registradas curvas de força versus distância de deflexão no carregamento e no descarregamento. Os valores de força no descarregamento a $3 \mathrm{~mm}, 2 \mathrm{~mm}, 1 \mathrm{~mm}$ e $0,5 \mathrm{~mm}$ foram tomados para comparação entre os grupos. Adicionalmente, foram realizados ensaios de tração em máquina universal de ensaios (Instron 5582, Canton, MA, EUA) para avaliar o comportamento mecânico dos fios em carregamento uniaxial. Os fios foram tracionados até uma deformação de $0,06 \mathrm{~mm} / \mathrm{mm}$ e então descarregados até que a carga fosse igual a zero.

O material como recebido foi analisado por DRX, seccionando-se fragmentos de 10 $\mathrm{mm}$ de comprimento e posicionando-os lado a lado de forma que pudessem ser caracterizadas as fases presentes no material. Os difratogramas foram obtidos utilizando radiação do tipo $\mathrm{Cu} \mathrm{Ka}(\lambda=0,15418 \mathrm{~nm})$ numa faixa de $30^{\circ}<2 \theta<100^{\circ}$ com passo de $0,02^{\circ} / \mathrm{s}$. As fases foram identificadas através de comparação com padrões disponíveis no banco de dados de estruturas cristalinas ICSD.

\section{RESULTADOS E DISCUSSÃO}

\section{1 - Microscopia eletrônica de varredura}

As médias dos resultados obtidos por EDS são resumidas na tabela 2. Todos os materiais estudados possuem leve excesso de níquel, o que sugere a presença de precipitados $\mathrm{Ti}_{3} \mathrm{Ni}_{4}$, que endurecem a liga, permitindo um aumento no limite de escoamento e limitando deformações plásticas a valores mais elevados de tensão, favorecendo o EMF e a SE. 
Tabela 2 - Composição em \% at. obtida por EDS.

\begin{tabular}{ccccccccc} 
& 3M SE & 3M SE & AO SE & AO SE & 3 M TA & 3 M TA & AO TA & AO TA \\
& 18 & 16 & 18 & 16 & 18 & 16 & 18 & 16 \\
\hline$\% \mathrm{Ni}$ & 50,6 & 50,7 & 50,9 & 51,0 & 51,6 & 51,5 & 50,6 & 50,9 \\
$\% \mathrm{Ti}$ & 49,4 & 49,3 & 49,1 & 49,0 & 48,4 & 48,5 & 49,4 & 49,1 \\
\hline
\end{tabular}

A análise das superfícies evidencia que os fios passam por algum tipo de acabamento superficial, uma vez que não se observa uma camada de óxidos de titânio na superfície. O tratamento superficial é desejável para a utilização do NiTi na área de ortodontia, uma vez que o atrito entre os bráquetes e o fio torna 0 tratamento menos eficaz. Observa-se nas figuras 2 e 3 as superfícies dos fios.

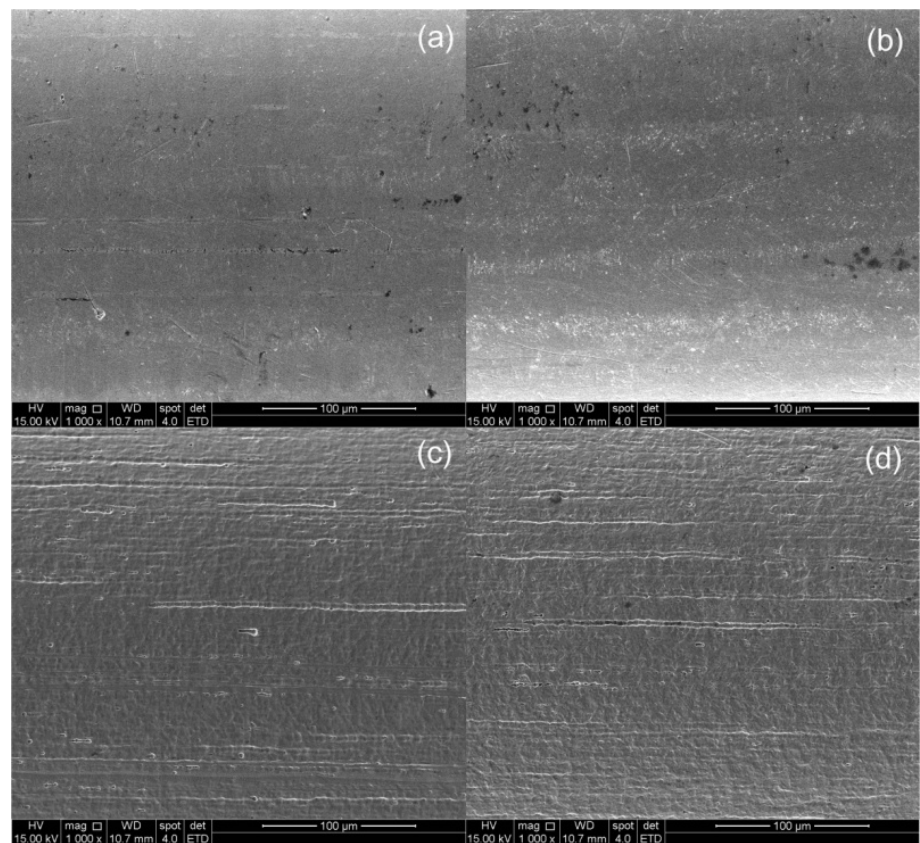

Figura 2 - Superfície dos fios (a) AO SE 18, (b) AO SE 16, (c) 3M SE 18 e (d) 3M SE 16

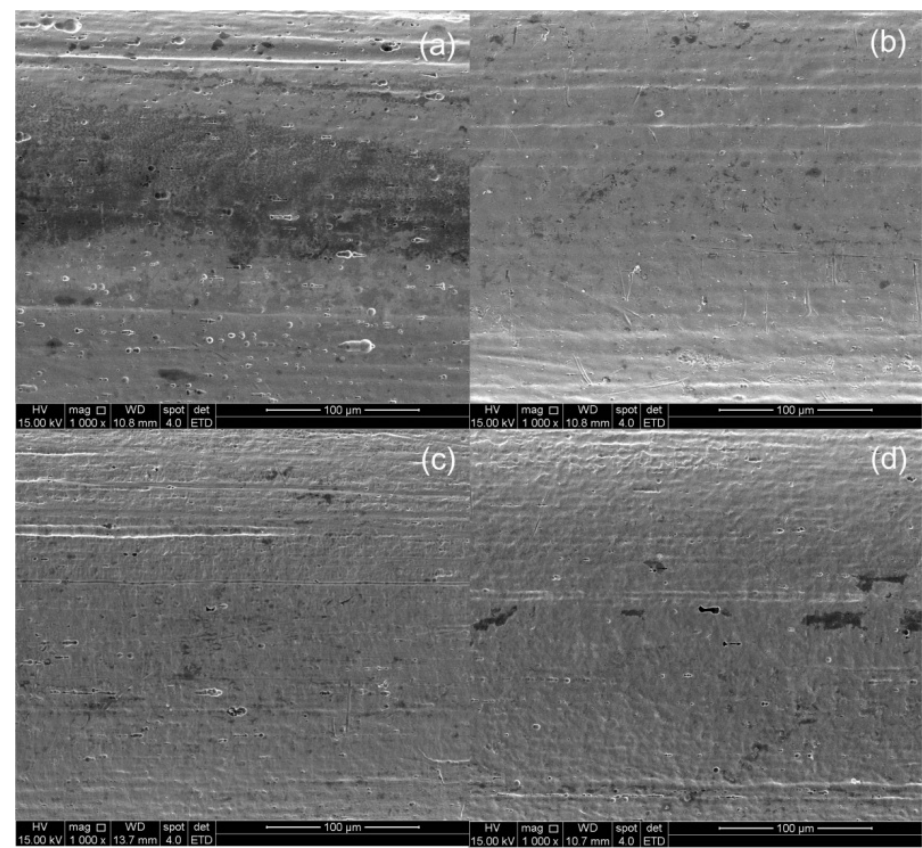

Figura 3 - Superfície dos fios (a) AO TA 18, (b) AO TA 16, (c) 3M TA 18 e (d) 3M TA 16. 


\section{2 - Caracterização mecânica}

Os ensaios de dobramento de três pontos exibem resultados característicos para este tipo de carregamento em NiTi. Os fios superelásticos apresentam recuperação total da forma quando se avaliam as curvas de força versus deflexão para este grupo. Os fios termoativados, por sua vez, apresentam uma deformação residual após o ensaio, o que está de acordo com o fato de apresentarem martensita induzida por tensão. A deformação nos testes de bancada ocorre a uma temperatura em que a fase martensítica induzida por tensão permaneça estável, sendo assim, não há condições para que haja recuperação de forma no descarregamento. As curvas de carregamento são apresentadas na figura 4 . A tabela 3 resume as forças obtidas em quatro pontos distintos do descarregamento.
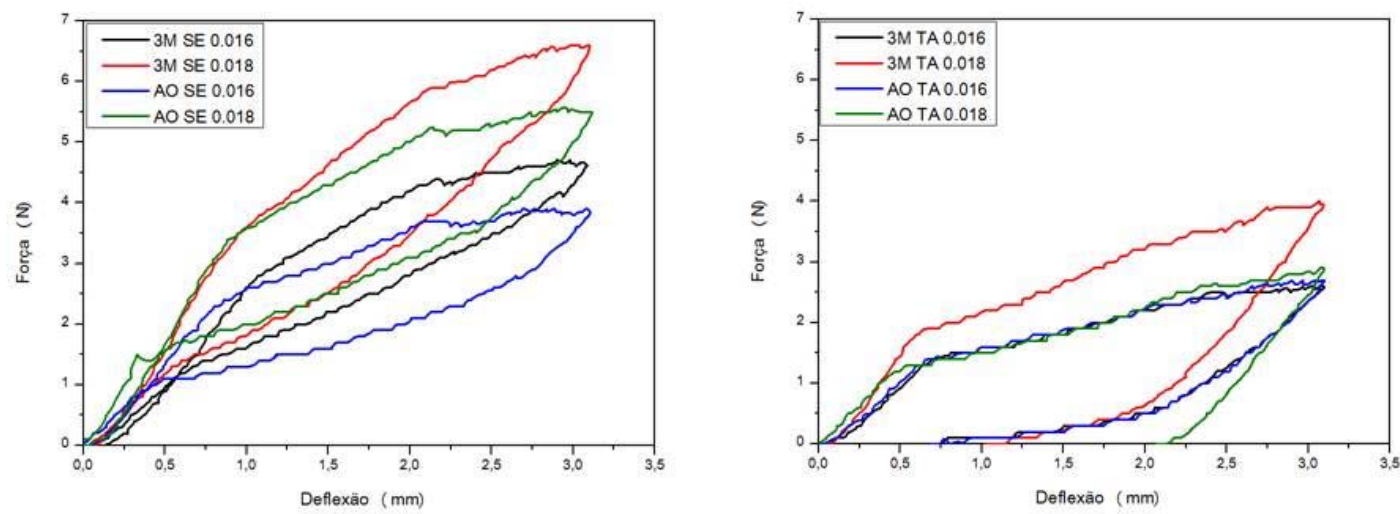

Figura 4 - Curvas de dobramento de três pontos dos fios SE (esquerda) e TA (direita).

Tabela 3 - Tabela de forças $(\mathrm{N})$ medidas a 3, 2, 1 e 0,5 mm de deflexão no descarregamento em dobramento.

\begin{tabular}{ccccc}
\hline Fios & $\mathbf{3 , 0} \mathbf{~ m m}$ & $\mathbf{2 , 0} \mathbf{~ m m}$ & $\mathbf{1 , 0} \mathbf{~ m m}$ & $\mathbf{0 , 5} \mathbf{~ m m}$ \\
\hline AO SE 18 & 5,0 & 3,1 & 2,0 & 1,6 \\
AO SE 16 & 3,5 & 2,1 & 1,3 & 1,1 \\
3M SE 18 & 6,1 & 3,5 & 1,8 & 1,2 \\
3M SE 16 & 4,3 & 2,8 & 1,6 & 1,0 \\
AO TA 18 & 2,4 & 0,0 & 0,0 & 0,0 \\
AO TA 16 & 2,4 & 0,5 & 0,1 & 0,0 \\
3M TA 18 & 3,5 & 0,1 & 0,1 & 0,0 \\
3M TA 16 & 2,5 & 0,6 & 0,2 & 0,1 \\
\hline
\end{tabular}

Foram realizados também ensaios de tração uniaxial para avaliar os distintos estágios do carregamento e descarregamento dos fios ortodônticos. Observa-se nas figuras 5 e 6 que houve uma recuperação total da deformação para as amostras superelásticas, assim como notado pelos ensaios de dobramento. Já para as amostras termoativadas, o perfil da curva se mostra condizente com as demais no carregamento, apenas diferindo na tensão de patamar. No descarregamento é possível perceber que há uma fração de deformação que se recupera pela transformação reversa, uma vez que se observa uma mudança da inclinação da curva na descarga. 


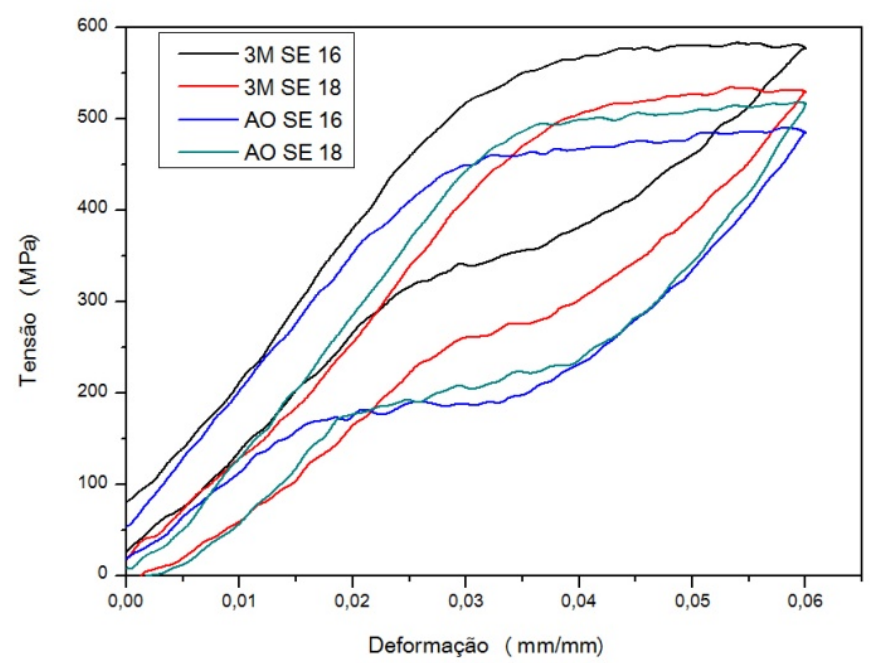

Figura 5 - Ciclo de carga e descarga em tração dos fios SE.

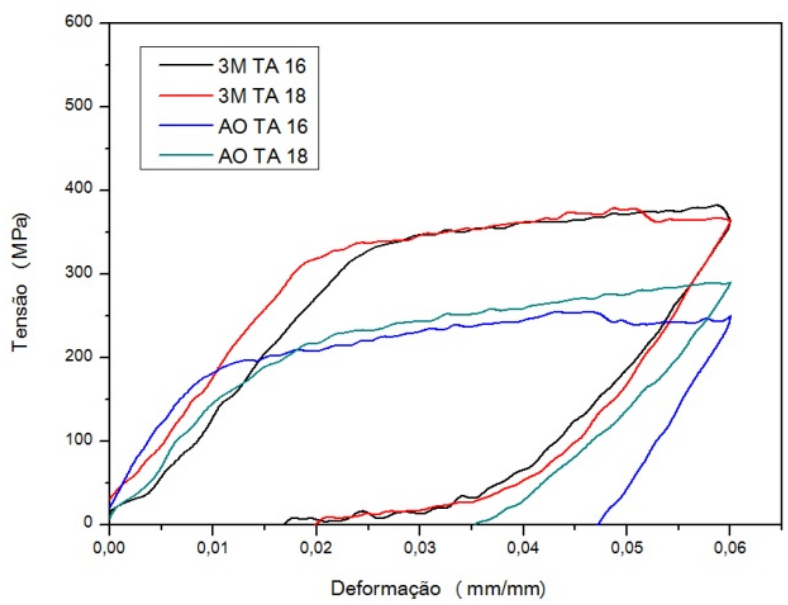

Figura 6 - Ciclo de carga e descarga em tração dos fios TA.

\section{3 - Difração de raios $X$}

Os difratogramas obtidos tanto para os fios superelásticos quanto para os fios termoativados, como recebidos, mostram apenas fase B2 na temperatura ambiente (figuras 7 e 8). O fato de que as curvas de carga e descarga em tração apresentarem um patamar de transformação reversa e uma deformação residual, relacionada à fração de martensita induzida por tensão, sugere que o material como recebido se encontra entre as temperaturas Af e As, em uma região de coexistência das fases martensítica e austenítica após o descarregamento, como descrito em [1]. Desta forma, é possível que o material apresente apenas a fase B2 em seu estado não carregado. 


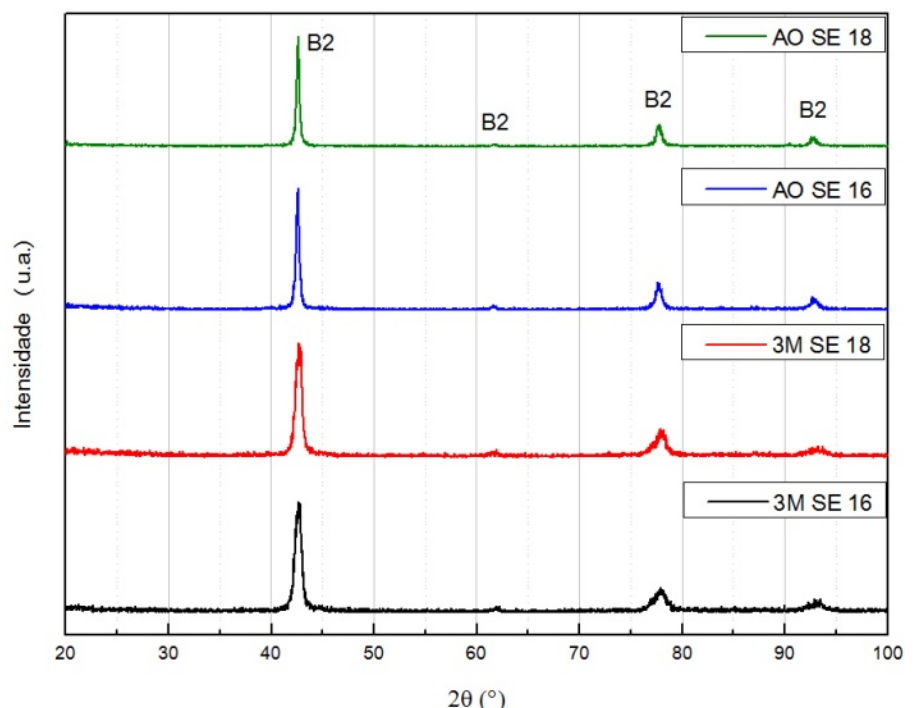

Figura 7 - Difratogramas obtidos para os fios SE.

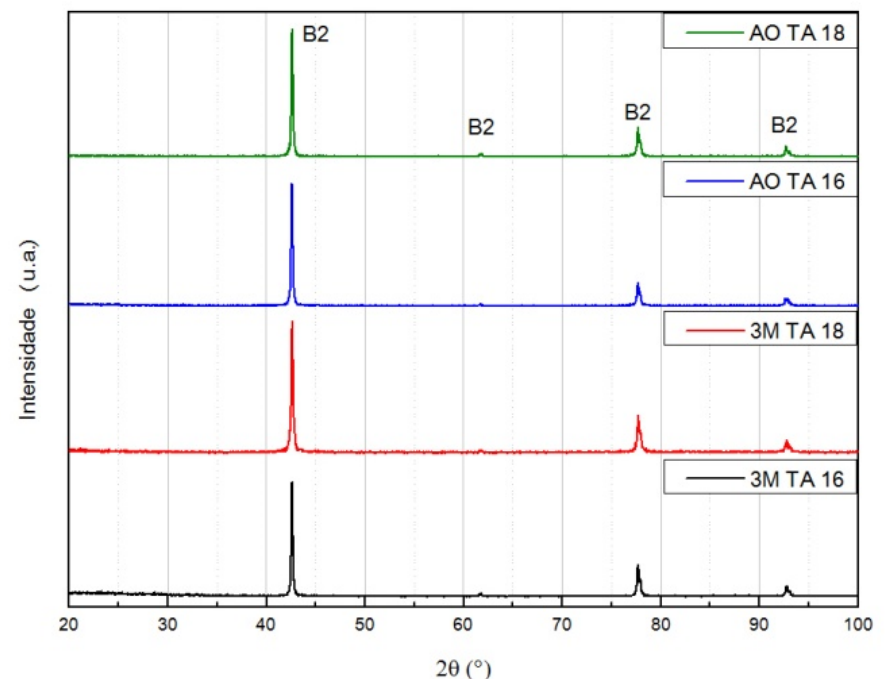

Figura 8 - Difratogramas obtidos para os fios TA.

\section{CONCLUSÃO}

Neste trabalho foi realizada a caracterização de fios ortodônticos fabricados a partir de NiTi. Os resultados obtidos levam a concluir que:

- Todos os fios, tanto superelásticos quanto termoativados apresentam apenas fase B2 quando se encontram na situação não tensionada;

- Há recuperação total de forma nos fios SE e parcial recuperação nos TA;

- Todos os fios termoativados apresentaram forças de descarga mais baixas quando comparados aos fios de mesmo fabricante e mesmo diâmetro do tipo superelástico;

- A composição de níquel em todos os fios foi superior a 50,5\%at., sugerindo a existência de precipitados que favorecem tanto SE quanto EMF;

- A superfície observada para os fios AO, tanto SE como TA, apresentam superfícies mais homogêneas, característica desejável para os tratamentos ortodônticos. 


\section{Agradecimentos}

Os autores agradecem ao CNPq, CAPES/PROEX, FAPEMIG e à PRPq/UFMG pelo auxílio financeiro.

\section{REFERÊNCIAS}

1 Otsuka K, Ren X. Physical metallurgy of Ti-Ni-based shape memory alloys. Progress in Materials Science. 2005; 50:511-678.

2 Hara T, Ohba T, Okunishi E, Otsuka K. Structural study of R-phase in Ti-50,23 at.\%Ni and Ti 47,75 at. \% Ni-1,50 at\%Fe alloys. Materials Transactions. 1997; 38:11-17.

3 Saburi T, Ti-Ni Shape memory alloys, in Otsuka K, Wayman CM. Shape Memory Materials. United Kingdom: Cambridge University Press. 1998; 49-96.

4 Kusy R. A review of contemporary archwires:Their properties and characteristics. The Angle Orthodontist. 1997; 67(3):197-207.

5 Nespoli A, Villa E, Bergo L, Rizzacasa A, Passaretti F. DSC and three-point bending test for the study of the thermomechanical history of NiTi and NiTi-based orthodontic archwires. Journal of Thermal Analysis and Calorimetry. 2015; 120:1129-1138. 